\title{
CORRELATION BETWEEN OVERACTIVE BLADDER (OAB) AND QUALITY OF LIFE (QOL).
}

\author{
V. Leanza1, S. Dati'2, A.A. Cavallaro'1, M. Bologna3 \\ 1 Obstetric and Gynecologic Department, Catania University (Italy) \\ 2 Casilino Policlinic Hospital Urogynecologic Unit, Rome (Italy) \\ 3 AO S. Camillo Forlanini, Rome (Italy)
}

\section{OBJECTIVE}

The aim of our study is to investigate the correlation between overactive bladder $(\mathrm{OAB})$ and quality of life (QL).

\section{METHODS OF STUDY SELECTION}

We studied articles concerning urinary incontinence (UI), overactive bladder $(O A B)$, and quality of life (QL). A review of the literature was undertaken using the Medline and Popline CD Rom, considering articles published from 1999 to 2008; additional sources were identified from references cited in relevant research articles. Data on incontinence, in addition to type, frequency, and amount of incontinence is reported. The correlation between QL and OAB was evaluated with the following validated questionnaires: Urgency Severity and Impact Questionnaire (USIQ), Urogenital Distress Inventory (UDI-6), Incontinence Impact Questionnaire (IIQ-7), Medical Outcomes Study Short Form-20 (SF20), Overactive Bladder Questionnaire (OAB-q), Medical Outcomes Study
(MOS), Epworth Sleepiness Scale (ESS), EuroQol 5-D (EQ-5D), Health related quality of life (HRQL), Work Productivity and Activity Impairment Questionnaire - Specific Health Problem (WPAI-SHP).

Data on incontinence, in addition to type, frequency, and amount of urinary symptoms is reported.

\section{RESULTS}

Women with $\mathrm{OAB}$ are also more likely to report a poorer quality of life. The negative effects of the $\mathrm{OAB}$ on self-efficacy were shown in several studies of health related behaviour. Symptoms of OAB (chronic frequency, urgency and urge incontinence, either alone or in any combination), are linked with depression and reduction of social activity. Interventions that are tailored to improve OAB symptomatology may improve depression and QL, but no permanent effective therapy has yet been found. Another aspect is the socio-economic consequence of the tiresome urge incontinence involving both the patient and the community. 


\section{CONCLUSION}

OAB symptoms are a tedious problem and have a significant impact on QL, affecting the social, psychological, physical and financial aspects of living both in women and men; the prevalence of urinary incontinence is significantly higher in women than in men. While urine loss itself is a disturbing symptom, older women are also likely to suffer from lower urinary tract symptoms associated with the overactive bladder syndrome such as urgency, frequency, mixed incontinence and nocturia. Nocturia was widely prevalent and increases with age, affecting men and women equally. Incremental increases in the number of voids/night have further negative effects on sleep, symptom bother, and HRQL. All these symptoms disrupt every day activities and interfere with general health status. Overactive bladder affects not only the older but also the young population (12\%). Overactive bladder symptoms reduce the QL more significantly than stress incontinence. Among the symptoms, urge and urinary incontinence were considered to be more related to patients' QL than frequency and nocturia. A common consequence of $\mathrm{OAB}$ is that the individual whose social self-efficacy is reduced tends to become depressed. Another aspect is socio-economic aspect of both the patient and the community. Until now, an efficacious overall permanent therapy to solve OAB symptoms has not been found.

\section{INTRODUCTION}

The primary symptoms of overactive bladder (OAB) include urinary urgency and frequency, with or without urge incontinence. OAB is urodynamically characterised by involuntary contractions of the detrusor muscles of the bladder. Despite the growing awareness of $\mathrm{OAB}$ as a chronic medical condition, little is known about the disease's economic burden.

Urodynamic diagnoses of detrusor overactivity, mixed incontinence, and stress incontinence with $\mathrm{OAB}$ are associated with significantly worse incontinence related bother and health related quality of life (QL) compared to those with stress incontinence without $\mathrm{OAB}[1,2,3]$.

There is a variance in epidemiological data regarding the prevalence rates of various urinary symptoms because of inconsistent definitions of incontinence, differences in questionnaires, settings, and methodology, as well as the reliability of self-report data [4-8]. Many questionnaires have been used to evaluate the QL, even if they have some limitations. Although these questionnaires are useful for comparing health-related quality of life (HRQL) among different diseases, they are often less sensitive for measuring specific aspects of a particular disease, compared to a condition or disease specific HRQL questionnaire. Attention has mainly focused on the consequences of symptoms linked to the overactive bladder. With the increasing interest in the overactive bladder, such information may add to understanding the problem and might 
be important for health-care decisionmaking [9]. Moreover the effect of self-efficacy on the QL has been investigated in several health related behaviour studies. Self-efficacy and QL are positively related while depression and self-efficacy are negatively related. The choices, goals, effort, and persistence of an individual can be affected by depression. Interventions that are tailored to increase self-efficacy may reduce discomfort and improve the patient's QL $[10,11]$.

The aim of our study is to investigate correlation between $\mathrm{OAB}$ and QL.

\section{METHODS OF STUDY SELECTION}

We studied articles concerning urinary incontinence (UI), OAB, and QL. A review of the literature was undertaken using the Medline and Popline CD Rom, considering articles published from 1999 to 2008; additional sources were identified from references cited in relevant research articles.

Data on any incontinence, in addition to type, frequency, and amount of incontinence is reported. Correlation between QL and OAB was evaluated with the following validated questionnaires: Urgency Severity and Impact Questionnaire (USIQ), Questionnaire; Urogenital Distress Inventory (UDI-6), Incontinence Impact Questionnaire (IIQ-7), Medical Outcomes Study Short Form-20 (SF-20), Overactive Bladder Questionnaire (OAB-q), Medical Outcomes Study (MOS), Epworth Sleepiness
Scale(ESS), EuroQol 5-D (EQ-5D), HRQL, Work Productivity and Activity Impairment Questionnaire - Specific Health Problem (WPAI-SHP).

\section{RESULTS}

Liberman et al. [12] conducted a survey in the United States among an age and sex-stratified sample of 4,896 non-institutionalised adults, 18 years of age and older. From the responses to the telephone survey, a total of 483 individuals with overactive bladder symptoms and 191 controls completed a mailed follow-up questionnaire to assess their QL using the Medical Outcomes Study Short-Form 20. After adjustment for age, sex, and the use of medical care, the greatest differences in the QL scores between patients with an incontinent $\mathrm{OAB}$ and controls were in the health perception (17.6 points; $\mathrm{P}<0.001$ ) and role functioning (13.0 points; $\mathrm{P}<0.001$ ) scales. Those with OAB, with frequency or urgency symptoms, or both, but without incontinence, also had significantly lower scores than did the controls in terms of mental health $(\mathrm{P}=0.026)$, health perception $(\mathrm{P}=0.01)$, and bodily pain $(\mathrm{P}=$ 0.016). These data indicate that individuals with $\mathrm{OAB}$ experience a reduction in their QL compared to community controls. An important new finding from this study is that individuals with $\mathrm{OAB}$, even without demonstrable urine loss, also have a poorer QL than that of controls.

In a study by Milsom et al., [13] $65 \%$ of men and $67 \%$ of women with 
OAB reported that their symptoms had an effect on their every day life , and $60 \%$ of those with symptoms found them bothersome enough to consult a medical practitioner. Frequency and urgency alone (59\%) were almost as common as urge incontinence (66\%) as reasons for seeking help. Of those who sought medical care, only $27 \%$ were receiving medication for symptoms at the time of the interview. Of those who were not taking medication, $27 \%$ had previously tried pharmacologic treatment, which failed. Of those who were not taking medication and who had never tried drugs, 54\% reportedthey were likely to discuss the problem with a physician again and $46 \%$ were not. Of those who had tried drugs but for whom the drugs had failed, 65\% reported they were likely to discuss the problem with a physician again and 35\% were not. Liberman et al. [14] assessed the impact of OAB symptoms on the QL in a community based US sample population. The survey was conducted in 2 phases: (1) a cross-sectional householdtelephone survey was performed among an age-stratified sample of 4,896 adults; and (2) a follow-up questionnaire was mailed to a subset of these respondents to assess their HRQL. The Medical Outcomes Study Short-Form (SF)-20 was used. This measures HRQL during the past month in 6 domains: physical functioning, role functioning, social functioning, mental health, health perception, and bodily pain. Both groups, wet $O A B$ and dry $O A B$, had significantly lower crude HRQL scores than the control groups in every domain. This was also true after adjustment for confounders. Statistically significant differences were observed in 5 of the 6 domains for the whole OAB group, in all 6 domains for the wet $\mathrm{OAB}$ group, and in 3 of 6 domains for the dry OAB group. In the dry OAB group, after adjustment for confounders, individuals with both frequency and urgency symptoms had statistically significantly lower scores than did the controls in all 6HRQL domains. There were numerical differences for the frequency-only and urgency-only subgroups, but these did not reach statistical significance. Individuals reporting more than 11 micturitions per day did have statistically significant lower domain-specific scores than those of controls in the areas of physical functioning, mental health, and bodily pain. The HRQL scores for individuals with 9 to 10 micturitions per day were not significantly different from those of controls. Abrams et al. [15] reported that patients with OAB were found to have a lower QL in the social and functional domains of SF-36 than did patients with diabetes. They pointed out that many patients with OAB tend to stop pursuing enjoyable social and physical activities, living with the condition in silence because they are too embarrassed to talk about their condition or are unaware that it can be treated. They list the most common reactions to the urinary incontinence component of OAB as embarrassment, frustration, anxiety, annoyance, depression, and fear of odour. Abrams et al. also enumerate the 
elaborate behaviour (coping mechanisms) that many patients develop, which are aimed at hiding and managing such urine loss. They also cite the results of Kelleher et al. [16] who used the King's Health Questionnaire, a survey originally developed to evaluate QL in women with urinary incontinence. Using this survey, women with $\mathrm{OAB}$ and incontinence were reported to have significantly greater QL impairment compared with women with stress incontinence and normal urodynamic function. These last few reports emphasize the difficulty in assessing impairment of QLin patients with OAB: most of these surveys have been performed in patients with wet $\mathrm{OAB}$, which must be acknowledged, because QL impairment in this group is most likely to be greater than in the dry OAB group. More recent studies have begun to overcome the deficit in data on QL as it applies to the whole OAB population and its subdivisions of wet $\mathrm{OAB}$ and dry $\mathrm{OAB}$.

Brown et al. [17] provide an overview of the impact of $\mathrm{OAB}$ on other problems now known to coexist with this condition. They report that urinary incontinence is independently associated with falls and fractures among community-dwelling, elderly women, in that women with weekly urge incontinence have a 26\% greater risk of sustaining a fall and a $34 \%$ increased risk of fracture after adjusting for other causes. More frequent incontinence was associated with increased risk, and women with daily urge incontinence had increased risks of $35 \%$ and $45 \%$ of sustaining falls and fractures, respectively. These investigators believe that because previous studies have demonstrated that urge incontinence has been associated with frequency/urgency and nocturia, OAB symptoms, and not just urge incontinence, have the potential to increase the risk of falls and fractures among elderly women.They cite previous studies as identifying urinary tract infections and skin infections as factors that increase the cost of $\mathrm{OAB}$ and cite recent analyses that suggest a potential reduction in health care costs for patients receiving treatment for $\mathrm{OAB}$. After the diagnosis of $\mathrm{OAB}$, the number of services received for urinary tract infections and skin infections decreased by $40 \%$ and 60\%, respectively, and was associated with potential cost savings in asmall sampling from the 1996 to 1997 California Medicaid programme. Regarding depression, Brown et al. cite data that suggest a strong association between depression and urge incontinence in a survey using a Beck Depression Inventory. They cite the prevalence of depression as 60\% in those with idiopathic urge incontinence, $42 \%$ in patients with mixed incontinence, and as only $14 \%$ in patients with stress incontinence.

Van Der et al., [18] found that the prevalence of stress incontinence (39\%), urge incontinence (15\%) and OAB symptoms (12\%) was also high in the young population. Compared with urge incontinence symptoms, OAB symptoms similarly reduced their QL, but stress incontinence did not significantly affect their QL. Women with OAB symptoms were es- 
pecially limited in their mobility, whereas urge incontinence was especially associated with feelings of embarrassment.

Coyne et., [19] al carried out a national survey in the USA to assess the prevalence of $\mathrm{OAB}$ and nocturia. A nested case-control study was conducted among respondents with $\mathrm{OAB}$ symptoms and age- and gendermatched controls, with participants completing a series of questionnaires on HRQL (OAB-q, Short Form-36, and MOS sleep scale). Descriptive analyses, t-tests, analysis of variance with post hoc comparisons and multivariate regressions were used to analyse the data. 5,204 people participated in the survey, with 919 in the nested case-control study. The sample population had a mean age of 45.8 years, was $52.6 \%$ female and $80 \%$ Caucasian. In the community sample, $31 \%$ reported $>1 \mathrm{void} / \mathrm{night}$ and $14.2 \%$ reported $>2$ voids/night. The prevalence of nocturia increased with age, with no gender differences. For $\mathrm{OAB}$ cases, $66.8 \%$ reported $>1$ void/night and $42.2 \%$ reported $>2$. In the case-control cohort there were significant HRQL differences $(\mathrm{P}<$ 0.01 ), with increasing episodes of nocturia in all OAB-q subscales except social interaction. The amount of sleep per night was significantly correlated with the sleep, concern and social interaction $\mathrm{OABq}$ subscale scores. The number of nocturia episodes/night was also significantly $(\mathrm{P}=0.02)$ associated with the number of hours of sleep/night.

Brunner et al., [20] evaluated the effect of OABsymptoms on women's
QL during and after their first pregnancy, using self-reported symptombased QL questionnaires. In a prospective cohort study, 474 women were asked to complete four self-reported questionnaires. Urogenital symptoms were assessed with the UDI and the IIQ. A women was considered to experience dry $\mathrm{OAB}$ if she replied positively to the following two questions: 'do you experience a strong feeling of urgency to empty your bladder?'; and 'do you experience frequent urination?'. A women was considered to experience wet $\mathrm{OAB}$ if she replied positively to all of the following questions: 'do you experience a strong feeling of urgency to empty your bladder?'; 'do you experience frequent urination?'; and 'do you experience urine leakage related to the feeling of urgency?'. In all, 344 (72.6\%) women who returned all four questionnaires were included in the analysis. After the first childbirth there was a rapid decline in the prevalence of dry OAB $(45.2 \%$ to $7.9 \%, \mathrm{P}<0.001)$. In pregnancy the prevalence of wet $\mathrm{OAB}$ increased significantly, but a year after childbirth the prevalence of wet $\mathrm{OAB}$ decreased and was similar to that at 12 weeks of gestation ( $\mathrm{P}=0.289)$. Women with wet $\mathrm{OAB}$ had higher scores in all IIQ domains than those with no OAB symptoms at 36 weeks of gestation. Women with either dry or wet OAB all had higher scores in the mobility domain than those with no OAB. The scores in the physical, social and emotional functioning domains were low, suggesting a minimal restriction of lifestyle. The conclusion was as 
follows : OAB symptoms are common during pregnancy; dry OAB had no negative effect on QL, whereas wet OAB compromised QL both during and after pregnancy, mainly in the 'mobility' and 'embarrassment' domains. The urge urinary incontinence symptom in wet $\mathrm{OAB}$ seems to profoundly compromise QL. Apparently, in young mothers with wet OAB, limitations in mobility are especially stressful and these symptoms can be embarrassing. Lowenstein et al., [21] developed a validated, patient-oriented questionnaire to assess urgency and associated life impact in OAB. Based on this data, they composed the first draft of our USIQ. A focus group of $\mathrm{OAB}$ patients provided feedback on the USIQ draft. The revised questionnaire has two parts: symptom severity (USIQ-S) and related quality of life (USIQ-QOL). The questionnaire was given to patients with clinically diagnosed OAB to establish face, content and discriminatory validity. Cronbach's alpha for the USIQ$\mathrm{S}$ and USIQ-QOL were 0.85 and 0.90, respectively. USIQ-QOL was moderately correlated with UDI-6 $(r=0.49$, $\mathrm{p}<0.001)$, IIQ-7 $(\mathrm{r}=0.77, \mathrm{p}<$ $0.001)$, and OAB-q ( $\mathrm{r}=0.73, \mathrm{p}<$ 0.001). Mean USIQ-QOL and USIQ-S scores differed by clinical diagnosis. The USIQ is an easily understood questionnaire with adequate validity for use in clinical practice and research. Coyne KS, Sexton CC et al., [22] examined the effect of OAB and other lower urinary tract symptoms (LUTS) on HRQL in a population sample, as OAB often occurs in conjunction with many other LUTS. A nested case-control analysis was performed on men and women with (cases) and without (controls) OAB, from the EPIC study. OAB was assessed using 2002 International Continence Society definitions. Based on their responses to questions about LUTS, the cases were classified into five groups; continent $\mathrm{OAB}, \mathrm{OAB}$ with incontinence, $\mathrm{OAB}+$ postmicturition, $\mathrm{OAB}+$ voiding, and $\mathrm{OAB}+$ postmicturition + voiding. Both cases and controls were asked questions about symptom bother (OAB-q), generic QL (EQ-5D), work productivity WPAI, depressive symptoms (Center for Epidemiologic Studies Depression Scale), sexual satisfaction, and erectile dysfunction (men only) using the Massachusetts Male Aging Study. Cases answered additional conditionspecific questions HRQL (OAB-q short form), Patient Perception of Bladder Condition and WPAI-SHP. General linear models were used to evaluate group differences. Of the EPIC participants, 1,434 identified OAB cases were matched by age, gender and country, with 1,434 participants designated as controls. Cases and controls were primarily Caucasian (96.2\% and $96.7 \%$, respectively), and most (65\%) were female; the mean age was 53.8 and 53.7 years, respectively. Comorbid conditions differed significantly by case/control status, with cases reporting significantly greater rates of chronic constipation, asthma, diabetes, high blood pressure, bladder or prostate cancer, neurological conditions and depression. There were significant differences between cases and controls in 
all reported LUTS. The OAB + postmicturition + voiding group reported significantly greater symptom bother, worse HRQL, higher rates of depression and decreased enjoyment of sexual activity, than the other subgroups. The conclusion was that $\mathrm{OAB}$ has a substantial, multidimensional impact on patients; OAB with additional LUTS has a greater impact. The diagnosis and treatment of OAB should be considered in conjunction with other LUTS, to improve not only one symptom but the QL of the human being.

\section{CONCLUSION}

OAB symptoms are a tedious problem and have a significant impact on QL, affecting the social, psychological, physical and financial aspects of living both in women and men; the prevalence of urinary incontinence is significantly higher in the former than in the latter. While urine loss itself is a disturbing symptom, older women are also likely to suffer from lower urinary tract symptoms associated with OAB such as urgency, frequency, mixed incontinence and nocturia. Nocturia was widely prevalent and increases with age, affecting men and women equally. Incremental increases in the number of voids/night have further negative ef- fects on sleep, symptom bother, and HRQL. All these symptoms disrupt daily activities and interfere with general health status.

OAB affects not only the elderly but also the young population (12\%). OAB symptoms reduce the QL more significantly than stress incontinence. Among the symptoms, urge and urinary incontinence were considered to be more related to patients' QL than frequency and nocturia. A common consequence of $\mathrm{OAB}$ is the tendency to depression of the individual who reduces his social self-efficacy. Then there is the socio-economic aspect for both the patient and the community. The socio-economic consequences of $\mathrm{OAB}$ have been estimated in a total cost of 12.6 billion US dollars per year.

Despite the prevalence of $\mathrm{OAB}$, many patients do not seek medical assistance for this condition because of embarrassment or the misconception that it represents an inevitable function of aging. Management includes implementing behavioural changes such as a reduced intake of caffeine, bladder and pelvic floor training, as well as antimuscarinic drug therapy and this may improve the QL. Nevertheless, compliance is often problematic and no effective permanent therapy has yet been found. 


\section{REFERENCES}

1. SHELTON BROOME B.A. :

Impact of urinary incontinence on self-efficacy and quality of life.

Health and Quality of Life Outcomes 2003, 1:35doi:10.1186/1477-7525-1-35.

2. NIPPON HINYOKIKA GAKKAI ZASSHI :

Economic impact of overactive bladder symptoms in Japan.

2008 Nov;99(7):713-22.

3. HAESSLER A.L., NGUYEN J.N., BHATIA N.N. :

Impact of urodynamic based incontinence diagnosis on quality of life in women. Neurourol Urodyn. 2008 Oct 30.

4. MOHIDE E.A. :

The prevalence and scope of urinary incontinence.

Clinics in Geriatric Medicine 1986, 2:639-655.

5. CONFERENCE NATIONAL INSTITUTES OF HEALTH URINARY

INCONTINENCE CONSENSUS :

Urinary incontinence in adults.

Journal of the American Medical Association 1989, 261:2685-2690.

6. RESNICK N., BECKETT .L, BRANCH L., SCHERR P., WETLE T. :

Short-term variability of self-report incontinence in older persons.

Journal of the American Geriatrics Society 1994, 42:202-207.

7. OUSLANDER J., SCHNELLE J. :

Incontinence in the nursing bome.

American College of Physicians 1995, 122:438-449.

8. OUSLANDER J., SCHNELLE J. :

Incontinence in the nursing bome.

American College of Physicians 1995, 122:438-449.

9. VAN DER VAART C.H., DE LEEUW J.R.J., ROOVERS J.P.W.R., HEINTZ A.P.M. : The effect of urinary incontinence and overactive bladder symptoms on quality of life in young women.

BJU International (2002), 90, 544-549.

10. BANDURA A. :

Self-efficacy: Toward a unifying theory of behavioral change.

Psychological Review 1977, 84:194-215. 
11. ZORN B.H., MONTGOMERY H., POEPER K., GRAY M., STEERS W.D. : Urinary incontinence and depression.

Journal of Urology 1999, 162:82-84.

12. LIBERMAN J.N., HUNT T.L., STEWART W.F., WEIN A., ZHOU Z., HERZOG A.R., LIPTON R.B., DIOKNO A.C. :

Health-related quality of life among adults with symptoms of overactive bladder: results from a U.S. community-based survey.

Urology. 2001 Jun;57(6):1044-50.

13. MILSOM I., ABRAMS P., CARDOZO L. et al. :

How widespread are the symptoms of an overactive bladder and bow are they managed? A population-based prevalence study.

BJU Intl 2001, 87: 760-766.

14. LIBERMAN J.N., HUNT T.L., STEWART W.F. et al. :

Health related quality of life among adults with symptoms of overactive bladder: results from a US community-based survey.

Urology 2001,57: 1044-1050.

15. ABRAMS P., KELLEHER C.J., KERR L.A. et al. : Overactive bladder significantly affects quality of life.

Am J Managed Care (suppl): 2000,S580-S590.

16. KELLEHER C.J., CARDOZO L.D., KHULLER V. et al. : A new questionnaire to assess the quality of life of urinary incontinent women.

Br J Obstet Gynaecol 1997,104: 1374-1379.

17. BROWN J.S., MCGHAN W.F., CHOKROVERTY S.A. : Comorbidities associated with overactive bladder.

Am J Managed. Care 6(suppl): 2001, S574-S579.

18. C.H. VAN DER VAART, J.R.J. DE LEEUW, J.P.W.R. ROOVERS, A.P.M. HEINTZ : The effect of urinary incontinence and overactive bladder symptoms on quality of life in young women.

BJU International 2002, 90, 544-549 544.

19. COYNE K.S., ZHOU Z., BHATTACHARYYA S.K., THOMPSON C.L., DHAWAN R., VERSI E. :

The prevalence of nocturia and its effect on bealth-related quality of life and sleep in a community sample in the USA.

BJU Int. 2004 Jul;94(1):194-54. 
20. VAN BRUMMEN H.J., BRUINSE H.W., VAN DE POL G., HEINTZ A.P., VAN DER VAART C.H. :

What is the effect of overactive bladder symptoms on woman's quality of life during and after first pregnancy?

BJU Int. 2006 Feb;97(2):296-300.

21. LOWENSTEIN L., FITZGERALD M.P., KENTON K., HATCHETT L., DURAZO-ARVIZU R., MUELLER E.R., GOLDMAN K., BRUBAKER L. : Evaluation of urgency in women, with a validated Urgency, Severity and Impact Questionnaire (USIQ).

Int Urogynecol J Pelvic Floor Dysfunct. 2008 Nov 20.

22. COYNE K..S, SEXTON C.C., IRWIN D.E., KOPP Z.S., KELLEHER C.J., MILSOM I. :

The impact of overactive bladder, incontinence and other lower urinary tract symptoms on quality of life, work productivity, sexuality and emotional wellbeing in men and women: results from the EPIC study.

BJU Int. 2008 Jun;101(11):1388-95. 